\section{AB0612 EFFICACY OF LOW DOSE AND SHORT-TERM IL-2 TREATMENT TO EXPAND ENDOGENOUS REGULATORY T CELLS IN PATIENTS WITH MIXED CONNECTIVE TISSUE DISEASE}

B. Zhang ${ }^{1}$, L.L. Shang ${ }^{1}$, J.P. $\mathrm{CaO}^{2}$, H.Y. Gao ${ }^{2}$, C. Gao ${ }^{3}$, X.F. Li ${ }^{2} .{ }^{1}$ The Second Hospital of Shanxi Medical University, Taiyuan, China; ${ }^{2}$ Rheumatology, The Second Hospital of Shanxi Medical University, Taiyuan, China; ${ }^{3}$ Brigham and Women's Hospital/Children's Hospital Boston, Harvard Medical School, Boston, American Samoa

Background: The concept of Mixed Connective Tissue Disease (MCTD) was first proposed by Sharp and his colleagues in $1972 .{ }^{1}$ MCTD has overlapping features between at least two autoimmune diseases, such as SLE, SSc, PM/DM and RA. Regulatory T cells (Treg cells) play a critical role in the maintenance of immune tolerance and the regulation of immune responses. Treg cell dysfunction contributes to the initiation of pathogenesis and the maintenance of high disease activity in RA and SLE. ${ }^{2}$ Recently, some studies have indicated that the proportion of Treg cells was different from healthy controls in SSc patients. ${ }^{3}$ Therefore, MCTD may be associated with Treg cells.

Objectives: To explore the status of CD4+T cells in peripheral blood of patients with MCTD; to explore the effect of low dose interleukin -2 (IL-2) treatment on the balance of Treg cells and Th17 cells in patients with MCTD, and to observe the short-term curative effect.

Methods: CD4+T cells in the peripheral blood of 58 MCTD patients and 33 healthy controls were analyzed by flow cytometry. All of 58 cases were treated with standard therapy, including corticosteroids, NSAIDs, immunosuppressants, or combination therapy. Among them, 26 cases of MCTD without using immunosuppressant (CYC, MTX, LEF, MMF), were as untreated group, another 32 cases were as treated group. Then we compared the difference of CD4+T cells between two groups. In 58 patients with MCTD, a total of 27 patients were treated with low-dose IL-2 (50WIU) on the basis of standard treatment for 5 days. The difference of CD4+T cells before and after treatment with IL-2 was compared.

Results: The absolute count of CD4+CD25+FOXP3+Treg cells in peripheral blood of patients with MCTD was significantly lower than those in healthy controls. There was no significant difference in the absolute count of CD4+CD25+FOXP3+Treg cells in the peripheral blood between the treated group and the untreated group. The absolute count of CD4+CD25+FOXP3+Treg cells was significantly higher than those before treatment with IL-2.

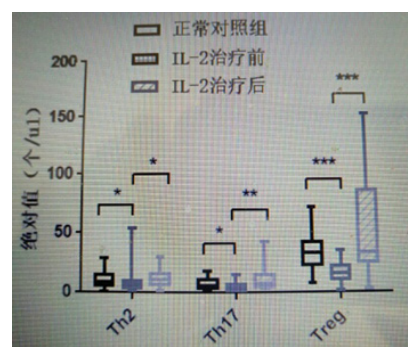

Conclusions: The absolute count of CD4+CD25+FOXP3+Treg cells in peripheral blood of patients with MCTD were significantly reduced, which may be the mechanism of immune imbalance in MCTD patients. The decrease of the absolute count of CD4+CD25+FOXP3+Treg cells in peripheral blood of patients with MCTD was not associated with the use of immunosuppressive agents. Low dose and short-term IL-2 treatment may increase the absolute count of CD4+CD25+FOXP3+Treg cells in the peripheral blood in patients with MCTD, so as to restore the immune balance in patients with MCTD.

References:

[1] Sharp GC, Irvin WS, Tan EM, et al. Mixed connective tissue disease - an apparently distinct rheumatic disease syndrome associated with a specifi c antibody to an extractable nuclear antigen (ENA). Am J Med 1972; 52: 148-159.

[2] Vila J, Isaacs JD, Anderson AE. Regulatory T cells and autoimmunity. Curr Opin Hematol, 2009, 16(4):274-279.

[3] Cordiali-Fei P, Mussi A, D'Agosto G, et al. Assessment of T regulatory cells and expanded profiling of autoantibodies may offer novel biomarkers for the clinical management of systemic sclerosis and undifferentiated connective tissue disease. Clin Dev Immunol, 2013, 2013:390563.

Acknowledgements: Thanks to my supervisor, my colleagues and superiors. Disclosure of Interest: None declared

DOI: 10.1136/annrheumdis-2017-eular.4017

\section{AB0613 PULMONARY ARTERY DIAMETER AND PULMONARY HYPERTENSION IN SYSTEMIC SCLEROSIS}

B.E. Schreiber ${ }^{1}$, C.P. Denton ${ }^{2}$, G. Robinson ${ }^{3}$, A.U. Wells ${ }^{4}$, G. Keir ${ }^{4}$, N. Sverzelatti ${ }^{5}$, J. Suntharalingam ${ }^{3}$, G. Coghlan ${ }^{1} .{ }^{1}$ Royal Free Hampstead NHS Trust: ${ }^{2}$ University College London, London: ${ }^{3}$ Royal United Hospital, Bath: ${ }^{4}$ Royal Brompton Hospital, London, United Kingdom; ${ }^{5}$ Parma University Hospital, Parma, Italy

Background: Systemic sclerosis may be complicated by pulmonary hypertension.
CT-determined pulmonary artery diameter has been shown to correlate with presence of pulmonary hypertension in idiopathic patients and in interstitial lung disease but not in scleroderma.

Objectives: To determine the utility of CT-determined main pulmonary artery diameter (MPAD) for predicting pulmonary hypertension (PH) in 97 patients with systemic sclerosis who had CT chest within six months of right heart catheterisation.

Methods: Retrospective review of right heart catheter and CT data done within six months in 97 patients. The CT scans were blindly scored by two radiologists. Results: 97 patients (75 female, 22 male) with systemic sclerosis who underwent $\mathrm{CT}$ scan and right heart catheterization were analysed. About $2 / 3$ had the limited subtype (64 limited, 24 diffuse, 6 undefined).

Pulmonary hypertension was present in 55 patients $(57 \%)$ and interstitial lung disease affecting $20 \%$ of the lung or more was present in 42 patients $(43 \%)$.

Survival was worse in those with pulmonary hypertension. Median survival was 9.3 years in those without pulmonary hypertension (8.1-10.5) and 4.4 years in those with pulmonary hypertension (2.6-6.2). Presence of interstitial lung disease and pulmonary hypertension were independent predictors of survival.

An ROC curve shows area under the curve of 0.864 . MPAD threshold of $25 \mathrm{~mm}$ or greater has a sensitivity of $98 \%$ and a specificity of $33 \%$ for the presence of pulmonary hypertension; $30 \mathrm{~mm}$ has a sensitivity of $73 \%$ and a specificity of $81 \%$ for the presence of pulmonary hypertension; $32 \mathrm{~mm}$ has a sensitivity of $56 \%$ and a specificity of $88 \%$ for the presence of pulmonary hypertension and $34 \mathrm{~mm}$ has a sensitivity of $35 \%$ and a specificity of $98 \%$ for the presence of pulmonary hypertension.

Survival was worse in those with a MPAD of $30 \mathrm{~mm}$ or greater, with median survival of 5.5 years (3.8-7.2) compared to survival of 9.3 years (7.9-10.7) in those with MPAD smaller than $30 \mathrm{~mm}$. It was not a significant predictor of survival after adjustment for presence of pulmonary hypertension and interstitial lung disease.

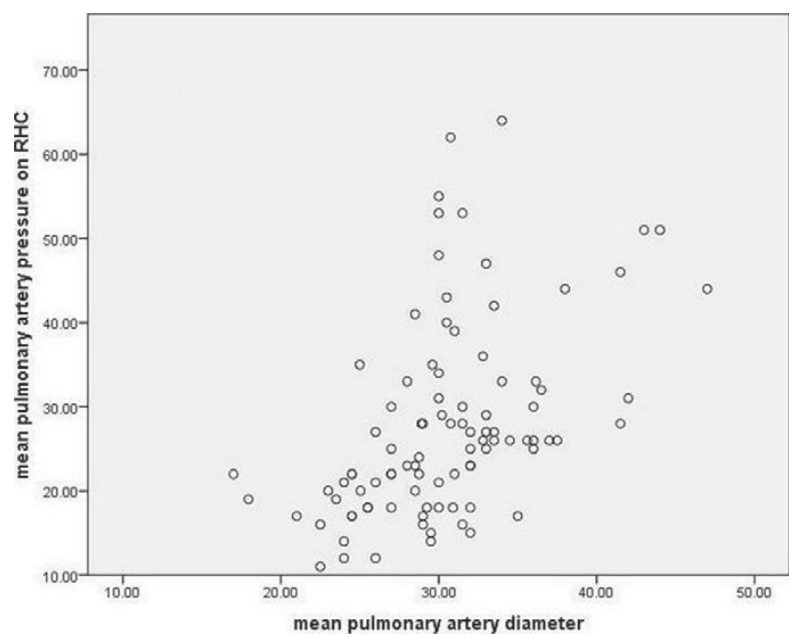

Conclusions: The CT determined MPAD correlates strongly with the presence of pulmonary hypertension in patients with systemic sclerosis.

Disclosure of Interest: None declared

DOI: 10.1136/annrheumdis-2017-eular.1872

\section{AB0614 ANTISYNTHETASE SYNDROME: AUTOANTIBODIES, CLINICAL PATTERN AND MANAGEMENT OF 17 SPANISH PATIENTS}

C. Busca, Á. Robles, I. Vives, J.A. Troncoso, L. Bailón, J.J. Ríos, A. Noblejas, F. Arnalich. Internal Medicine, la Paz Hospital, Madrid, Spain

Background: Antisynthethase syndrome (ASS) is a heterogeneous rare inflammatory condition characterized by myopathy, intersticial lung disease (ILD), arthritis, mechanic hands and Raynaud phenomenon (RP). The hallmark of ASS is the presence of antibodies against aminoacyl-transfer RNA synthetases (anti-ARS). Eight different anti-ARS have been described: anti-Jo1, anti-PL7, antiPL12, anti-EJ, anti-OJ, anti-KS, anti-Zo, anti-Tyr/YRS. Despite recent publications, correlation between autoantibodies clinical pattern and management in ASS is not entirely well-known.

Objectives: The aim of this study was to define clinical features, autoantibodies and outcomes of a series of ASS patients.

Methods: We retrospectively analyzed the epidemiology, clinical data, lung function parameters, muscle enzymes, electromyogram (EMG) and autoantibodies pattern and its relationship with clinical manifestations, treatment management and outcomes of 17 patients recruited between 2005 and 2016 from the Autoimmune Diseases Division of a Spanish hospital.

Results: A total of 17 patients were reviewed (15 female). Mean age at diagnosis was 55 years. Median time delay to diagnosis was 5 months. Median follow-up was 65 months. Main clinical symptoms were dyspnea (76.5\%), polyartrhitis $(58.6 \%)$, muscle weakness and myalgias $(52.9 \%)$, RP $(41.1 \%)$ and mechanic 